\title{
64
}

\section{A Real-Time distributed Multimedia virtual environment for Collaborative Engineering}

\author{
J.J. LaViola, R.J. Barton, G. Newton, and R. Cross \\ Fraunhofer Center for Research in Computer Graphics, Inc. \\ 321 S. Main St., Suite 2 \\ Providence, RI 02903 USA \\ (401) 863-6363 \\ \{jlaviola, rbarton, gnewton, rcross\}@crcg.edu
}

\begin{abstract}
In this paper we present the concept of Telepresence to describe an emerging and enabling communication medium. We define a Virtual Collaborative Environment (VCE) to exist when virtual reality and multimedia are combined to create a shared environment for human interaction and cooperation. We describe the fundamental problem with current attempts to evolve today's CAD software into a practical, shared, collaborative environment. We then present a new approach to the design of a shared virtual environment that addresses issues of scalability, networking, and synchronization, and discuss its applicability to collaborative engineering.
\end{abstract}

Keywords

Virtual collaborative environments, virtual reality, BARCO table, CAD

\section{INTRODUCTION}

\subsection{Background}

The rapidly developing information technology infrastructure is radically changing the methods and media by which people communicate and interact. As this evolution unfolds, human interactions will no longer be limited by the constraints of physical location, cultural and political barriers, or disabilities. Advanced computer graphics and high speed networking form the basis for what is now commonly called Telepresence. Telepresence describes a communications medium that enables a person to feel as if he or she is actually present in a different place or time. Virtual reality is an enabling technology that is described as 
immersive, interactive, and perceptively believable to the users' experience. The creation, delivery and reception of audio, video, text, and imagery data is defined as multimedia. Combining these technologies, we define a Virtual Collaborative Environment (VCE) to exist when multiple physically separated users share a distributed multimedia virtual environment for the purpose of sharing information or accomplishing a common objective. (Barton, Newton, 1997)

\subsection{Problems with existing CAD systems}

Existing industry standard $\mathrm{CAD}$ systems are inadequate and incomplete for a collaborative engineering environment because of three key areas, namely, pointto-point communication issues, network latency, and user interface technology.

Point-to-Point

Most implementations today are unicast in nature, not supporting many-to-many distributed users. Off -the-Shelf collaborative CAD today most often takes the form of shared data bases, shared $x$-windows, and comprehensive client server applications. A good example of this is the current release of AutoCAD v13c4, which uses the infrastructure of the World Wide Web and native browser software to share designs amongst distributed engineers. This model works well for distributed users, but is certainly not real-time collaboration.

\section{Latency}

There are several commercial off-the-shelf shared X-Windows type applications readily available on the market today. These systems are moderately successful on local high-speed networks, or LANs, i.e., 10 or 100 BaseT only, due to the high bandwidth required for acceptable performance. In order to realize a geographically distributed engineering environment, a practical solution must function in a high-latency environment. Currently, such a system does not exist. $\mathrm{TCP} / \mathrm{IP}$ is not an adequate transport mechanism for a real-time or near real-time multi-point environment.

\section{User interfaces}

The current state of user interface design is centered around the theme of onemachine, one-person interactions. This is inadequate for a collaborative engineering environment. New interfaces must be designed that are focused on groups of users, working together, sharing ideas on a single model or prototype in this environment. These new interfaces could be radical in nature, such as, immersion of the users and model in a shared Virtual Environment (VE) or using a projective display system such as the BARCO table, see Figure 1. Gesture recognition and visual coherency techniques such as motion blurring and ghosting can be integrated into an interface which mitigates problems associated with latency, as well as promotes collaboration amongst users. 
Figure 1 BARCO Table at CRCG.

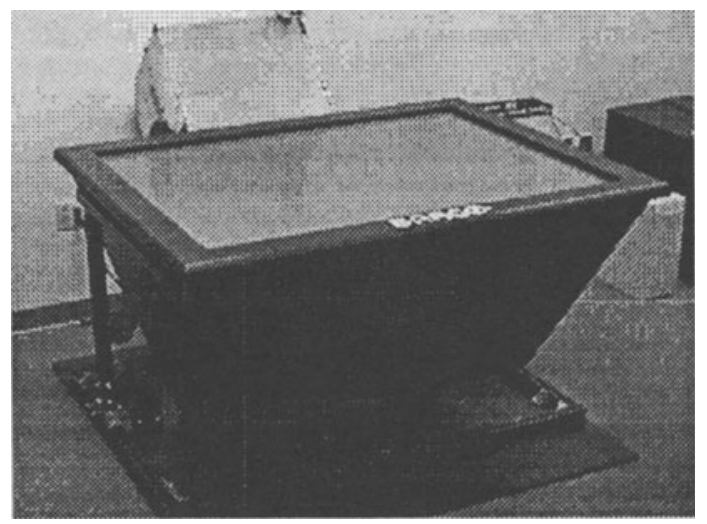

\subsection{Previous work}

Industry is currently evaluating various ways to capture the talent of their design and engineering personnel, without the need for physical co-location, in order to reduce cost, speed the design process, and remain competitive in the global economy. The design and review process must be collaborative, real-time, and distributed, often traversing great geographical distances and cultural boundaries.

The Shared 3D Viewer (Kress and Anderson, 1996), see Figure 2, is an application enabling a cooperative, distributed discussion of the three-dimensional geometry and product structure of CAD data. This tool is designed to support communication during the product development process particularly in the design phase. The innovative $3 \mathrm{D}$ functionality offers designers and project planners the proper format for the discussion of $\mathrm{CAD}$ data. Shared camera views, three-dimensional cursors and annotations allow the participants to discuss specific details of a product. An immersive experience is provided by stereo projection on either the desktop or BARCO table.

A set of 3D cursors can be used to direct interest at specific locations on the model. Cursors can be used to mark a point, length or area of the product, and are color coded according to the user that is controlling the cursor. Additional tools allow the measuring of lengths and angles within the three-dimensional window. Text messages can also be placed in the $3 \mathrm{D}$ scene, expanding the communication possibilities. In addition to the synchronous communication offered by the $3 \mathrm{D}$ cursors, the Shared 3D Viewer allows the creation of 3D annotations for asynchronous communication. Text, audio, and video messages can be created and attached to the CAD model so that users in later sessions can view the annotations. Three-dimensional icons represent the location and type of the annotation data. Through the use of MIME types, additional formats of annotation data can also be supported. 


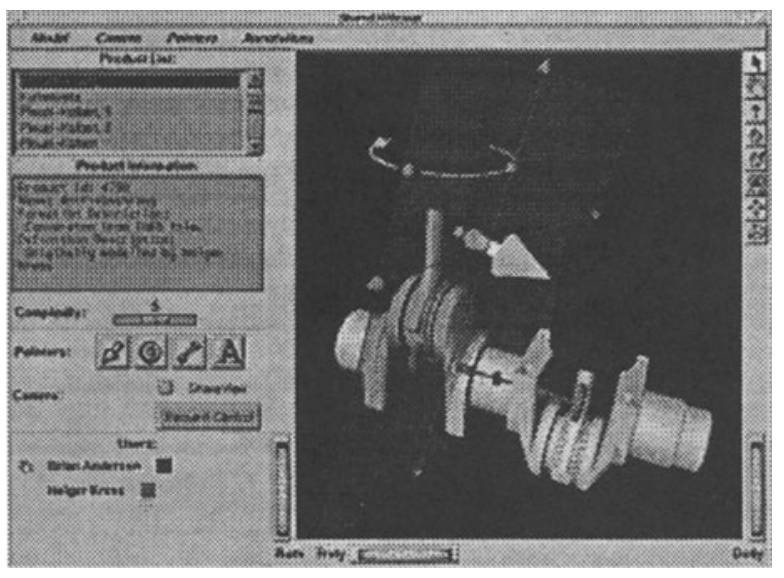

Figure 2: HP 3D Shared Viewer.

\subsection{Use of VCEs to support collaboration}

Effective communication is vital to nearly all aspects of business, yet the tendency towards global enterprise is making in-person communication harder. Face to face meetings are often prohibitively expensive, not only because of the cost of travel but also because of the valuable time lost while traveling. Face to face meetings involve multiple modes of communication which can not be duplicated by any ubiquitous communication device. The attendees can not only hear each other, they can also see each other and can interact with other objects together (e.g. a blueprint, a design prototype). Telephone conference calls allow people to speak to each other but do not permit any non-verbal communication. Video conferencing goes one step further and restores some of the missing non-verbal communication by allowing the participants to see live video of each other's faces, yet they are still unable to interact in any other way. Virtual teleconferencing provides participants with a VCE in which they can hold a virtual meeting. The multimedia aspects of the VCE allow the insertion of audio and video streams into the virtual environment, providing the participants with the same audio/video exchange capabilities found in video conferencing. However, the media streams are not limited to audio and video capture of the participants. Text, images, sound clips and video clips can also be embedded in the environment.

Virtual objects of interest (e.g. a 3D model of a design prototype) can be placed in the environment for the participants to view, interact with and discuss. A further advantage of virtual reality is that the virtual space can be divided into "rooms" as a way of organizing information that humans find intuitive (Card, 1991). 


\section{A SYSTEM ARCHITECTURE FOR VCE'S}

\subsection{Multiple network model}

The architecture outlined here is based on the observation that different VCE components have very different networking and synchronization requirements. Past attempts at mapping these disparate requirements to a single networking protocol have failed to produce solutions scalable to wide area general purpose networks. However, protocols currently exist to implement most, if not all, of these components individually. We have identified five classes of networked data which must be supported in order to provide a general purpose VCE architecture. These classes are shown in Table 1.

\begin{tabular}{l|l|l} 
Class & Example Data & Requirements \\
\hline Renderer & $\begin{array}{l}\text { Synchronization } \\
\text { between } \\
\text { renderers }\end{array}$ & $\begin{array}{l}\text { Lowest } \\
\text { latency }\end{array}$ \\
\hline Agent & $\begin{array}{l}\text { Communication } \\
\text { between agents }\end{array}$ & $\begin{array}{l}\text { Guaranteed } \\
\text { delivery, } \\
\text { flexibility }\end{array}$ \\
\hline Simulation & $\begin{array}{l}\text { Distributed } \\
\text { shared world } \\
\text { state updates }\end{array}$ & $\begin{array}{l}\text { Low latency, } \\
\text { variable QOS }\end{array}$ \\
\hline Device & $\begin{array}{l}\text { Input/Output } \\
\text { device events }\end{array}$ & $\begin{array}{l}\text { Low latency, } \\
\text { LAN use only }\end{array}$ \\
\hline Live Media & $\begin{array}{l}\text { Video textures, } \\
\text { live audio }\end{array}$ & $\begin{array}{l}\text { Like Internet } \\
\text { conferencing }\end{array}$
\end{tabular}

Table 1 Classes of Data

The architecture is based on assigning each class of data its own network. Potentially, several of these virtual networks can be implemented using the same network transport protocol. However, at this time, we anticipate using a different protocol for each class.

\subsection{Component model}

Each component of a VCE is allowed to participate in any set of these networks. The networks do not form a protocol stack, but rather parallel communication channels with different characteristics. Figure 3 shows an example set of components connected by the five networks. In the figure, each vertical cylinder represents a component. These components can be implemented as separate processes running on a set of networked computers. Components become clients of the various networks through a protocol-independent API represented by the five clouds in Figure 3. Because this interface is independent of the network protocol choices, this architecture will allow our research groups to research and develop better solutions for each class separately. The implementation section below expands on these protocol choices and possibilities. 


\section{Inter-Component communication}

The different "Networks" present a shared data abstraction to the components, which is accessed through a well defined API. Communication between components is permitted only in so far as multiple components can access the same objects in the shared data. Component developers therefore do not need to be aware of communication implementation details. The use of an API for intercomponent communication allows local communication as well as remote communication in a transparent manner. When multiple components are operating on the same node in the physical network, then their communication can be via shared memory rather than network packets. In the trivial case, a monolithic highperformance VE application running on a single machine can be assembled from reusable, networkable components. In this case, only shared memory communication would take place, providing a clean separation between components without generating network traffic. The API presented by each network is based on asynchronous sampling of the states of the objects that occupy the given network. Since components sample the various networks asynchronously, they can operate in a decoupled manner. Decoupled operation implies that components can operate independently of other components. This is an important characteristic of any VE design, because it allows rendering components to operate at a constant frame rate, independently of the current update rate of simulation components. The well-defined inter-component communication mechanism also allows different components to run on different processors, when available. In such a case, parallelization and pipelining of rendering, simulation, agent operation and device handling is possible.

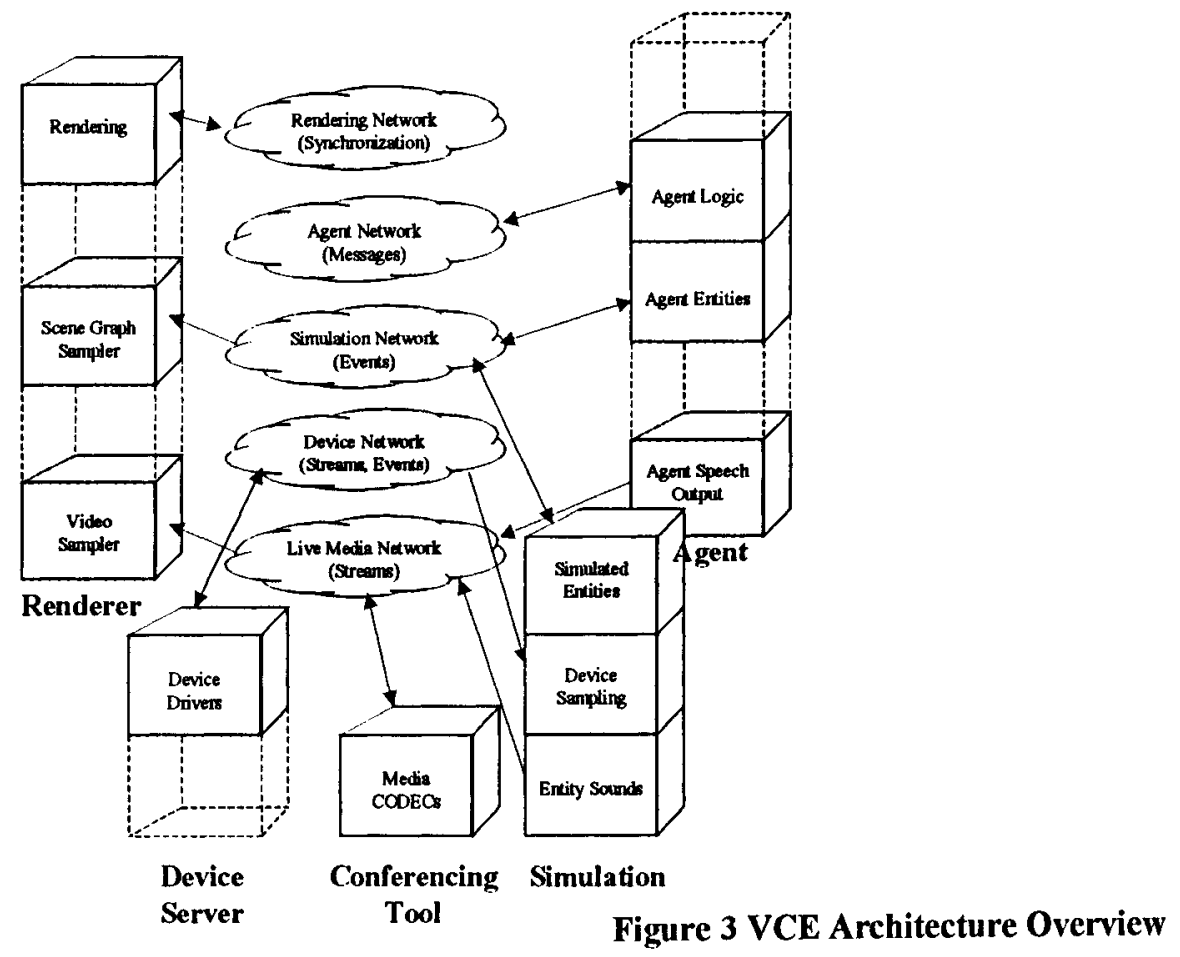




\subsection{Simulation component}

Note that some components, for example a simulation server, need to participate in only one type of network. The Simulation Network represents a shared world which is consistent even in the absence of the other networks. Simulations can join the network, explore the shared world and affect entities within it. However, they need not be concerned with issues of rendering or video conferencing. This will allow developers of simulation modules to remain unaffected by development concentrating on other areas of VCE. It also allows simulation-intensive virtual worlds to make use of computational resources which do not have graphics capabilities, such as server machines. We envision a typical virtual world utilizing multiple servers which provide the simulation for automated entities, while the computers used to display the various participants' views would run only very limited amounts of simulation, such as the individual user's avatar simulation.

\subsection{Rendering component}

\section{Live media rendering}

In order to provide spatial audio and video texture mapping, renderers for both graphical views and audio must have access to the Live Media Network. Stored audio effects as well as live network streams from remote dynamic sound sources, such as the voices of participants, have to be processed by a spatial audio renderer locally. The positions of the sound sources are known to the renderer since they are entities in the simulated world, which is being sampled by the renderer. The rendering of texture mapped video can be supported in the same way. Live video streams from the Live Media Network, as well as locally stored video clips, can be accessed by the renderer. The video streams are then sampled into texture memory and used as texture maps during rendering of the views. Fraunhofer CRCG's virtual teleconferencing application shown at ACM '97 demonstrated this capability.

\section{Sampling of the simulation network}

To allow the renderer to sustain a near-constant frame rate, it must operate independent of the simulation components. Such a decoupling is vital to the interactive experience (Pausch, 1994). We define the interface to the Simulation Network in such a way, that the renderer or other clients can easily sample the states of entities at specific points in simulated time. The client can construct a consistent snapshot of the world, which is then used to update the scene graph and rendered.

\subsection{Live media components}

The purpose of the Live Media network is to carry low-latency traffic such as audio and video streams. This type of traffic usually enjoys no quality of service assurances. Algorithms from Internet Telephony can be used on top of a multicast 
network infrastructure to implement the transport of these data types independently of the other types of information in the CVE system. Consequently, conferencing components could choose to participate just in the Live Media network. This could allow participants using non-VE systems, such as videophones, to join the collaborative world via only live media. Conforming to standards for video and audio encoding, such as G.728 and H.261, will ensure such interoperability. CRCG has demonstrated this capability with the VETAF system (Barton, 1997).

\subsection{Device components}

The purpose of the device network is to allow very high frequency and low latency connections between Virtual Reality hardware devices and the simulations that use these devices. In general, this network conists of devices connected locally or on the LAN. Connections on this network are usually only the polling or event processing of an input device to control a parameter in a simulation. However, combinations of input and output devices, such as haptic feedback devices coupled with trackers, may require direct connections between devices at the device frequency, potentially much higher than the simulation frequency. In order to support the highest possible fidelity, such direct inter-device connections will be implemented at the Device Network level and will not involve the simulation components.

\section{INTERFACE ISSUES}

\subsection{Motivation}

The use of the BARCO Table as our display device has numerous advantages in the collaborative engineering environment. The table is large enough to provide room for several participants to work together on the same physical display. Also, a group of networked tables allows collaboration locally as well as across intercontinental networks. The use of the table does present some humancomputer interaction problems such as:

- Single machine, several users - who grabbed the object first?

- Who has priority in a conflict situation?

- How do we minimize the effects of latency?

- How do we let users know that objects are in the control of another user?

- How do we provide a natural interface for creating and manipulating objects?

All of these issues will be addressed in the following sections.

\subsection{Locking and control}

In order to have a stable collaborative engineering environment, users must be able to create and manipulate objects easily and in an orderly fashion. The idea of 
object manipulation and ownership in a VCE is known as floor control. A distributed collaborative environment requires a strong mechanism to provide concurrency and floor control for its included objects. Model movements, attribute alterations, and other object property manipulation must be tightly controlled, without being centralized. Centralization reduces scalability and performance. The VCE developed for this project includes a distributed signal-locking device for object data in this environment. A site will make a request to the group for a specific key, which would then be granted in the event that it was not already in use. In the event that a key is in use, the requester would receive the key in turn, as it is released from other members of the group. In reference to the discussion of signaling protocols earlier, this type of data would not necessarily require near real time delivery to the group, but would require a guarantee of delivery. (Bukowski, 1995)

\subsection{Latency issues}

In order to realize a geographically distributed collaborative environment, a practical solution system must function in a high-latency environment. (Macedonia, 1994) High latency performance results in "popping" or "jerking" motions of graphical objects within the environment. One method for minimizing the negative impact of latency on the user interface is to apply what is known as visual coherency techniques. Our system includes advanced rendering techniques that address the latency problem, to include motion blurring and transparency, "ghosting".

Currently, known blurring algorithms are far too slow for the 3D interactive environment. A novel approach to blurring for $3 \mathrm{D}$ objects gives real time performance and demonstrates smooth and visually consistent motion. Besides looking more natural, motion blur establishes visual coherence between temporarily disparate renderings of the same object. This visual coherency is often disregarded in rendering, replaced instead with "popping" or "iconifying" of the object. Loss of coherency degrades the user's ability to synthesize meaningful actions occurring in the environment. Motion blur also assists interaction with moving objects in the virtual world. Blurring fast-moving objects simplifies the user's task of intercepting the object. By minimizing the strobing effect of motion and adding trails to the object, the user is better able to predict its motion path and velocity changes. Thus, the incorporation of motion blur enhances the natural rendition of the 3D environment to its participants, 3D interfaces become less confusing, and interaction with fast moving objects becomes easier. (Zeleznik, $1995,1996 a)$

Similarly, transparency is useful in a shared CAD environment. Ghost locking makes the user aware of many aspects of manipulating a shared object in a networked environment. The user has an immediate response to his or her actions, affording a feeling of control over the environment. The user also sees immediately 
that lag is occurring, because of the appearance of a "partial" or transparent object. Finally, fading out the copy that lost the lock gives the user time to understand the changed state of the manipulated object. (Conner, 1997)

This effect can be customized by varying levels of opacity in either the grabbed object or the original object, depending on outside knowledge about the state of the lock or the likelihood that the lock will be taken successfully. For example, a lock is more likely to fail for a highly contended object. In this case, the grabbed object can be more transparent, while the remaining object is more opaque, showing the user the likely results of trying to grab a highly contended object. (Conner, 1997)

\subsection{Gesture recognition}

Gesture-based interfaces offer an alternative to traditional keyboard, menu, and direct manipulation interfaces. The ability to specify objects, an operation, and additional parameters with a single intuitive gesture appeals to both novice and experienced users. Gesture-based interfaces for platforms such as the BARCO table are desirable, as they permit far more natural interaction than traditional mouse and keyboard interfaces. Our system includes gesture recognition techniques developed for SKETCH, a rapid virtual prototyping environment, developed at Brown University (Zeleznik, 1996b). In the early stages of a design, often what is required is a rapid conceptual model of the desired product. SKETCH is designed to bridge the gap between hand sketches and computer-based modeling programs, combining some of the features of pencil-and-paper sketching and some of the features of CAD systems to provide a lightweight, gesture-based interface to "approximate" 3D polyhedral modeling. SKETCH uses a gestural mode of input in which all operations are available directly in the $3 \mathrm{D}$ scene through a threebutton mouse or three-button stylus.

\section{Figure 4: Annotation of a Supertool}

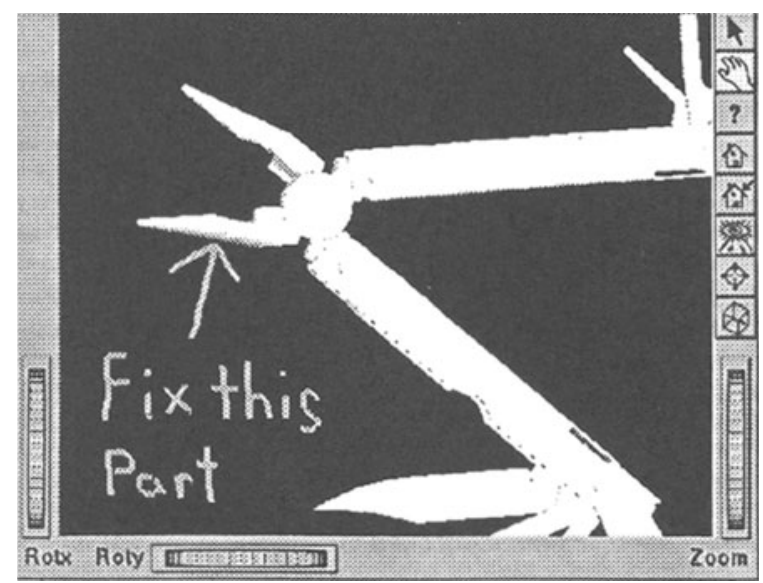




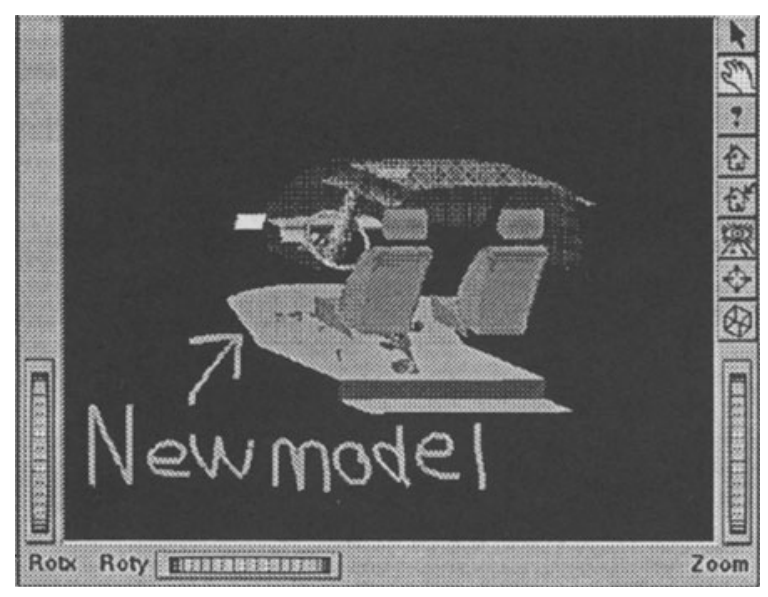

Figure 5: Annotation of a van design

The problem with the SKETCH interface is that it does not handle placing freeformed gestures at arbitrary locations in 3 space. It is useful if users can make free-formed gestures for annotation purposes so a particular piece of geometry can be referenced. This feature is good for design reviews or brainstorming. These free-formed gestures are placed on a $2 \mathrm{D}$ plane close to the film plane. They then must be transformed to point to an object in the 3D world. This is a very difficult task because a lot of guesswork is involved in determining where the user actually wants the gesture to be placed. Figures 4 and 5 show some snap shots of freeformed gestures used to annotate a model. If the user was to rotate the objects using a trackball, the annotations would stay in place in relationship to the $3 \mathrm{D}$ location that the annotation is pointing to. Using these annotations, managers can let engineers know what they think of designs, and make comments so communication can be enhanced.

Static hand posture recognition is currently the most common and widely used method for interaction involving glove based input devices. In order improve the naturalness of interaction, and thereby decrease the user-interface learning time, there is a need to be able to recognize dynamic gestures. Fraunhofer IGD has recently demonstrated a camera based tracking technique. This research will be integrated into a later version of our VCE.

\section{HAPTICS}

Performing detailed manipulative tasks in a collaborative virtual environment is a significant challenge. Virtual environments tend to address only the human visual system, ignoring the other senses. Since many interactions in virtual environments tend to involve direct manipulation (i.e., grabbing an object), the lack of tactile perception is particularly noticed. The addition of haptic feedback systems has 
proven to enhance understanding and performance in virtual environments. (Brooks, 1990) Our system will address the issues involved in adding haptic feedback to non-spatially immersive environments with the BARCO table.

Furthermore, it will explore techniques and user interface design for tactile vision substitution in the form of spatial, interactive haptic, tactile force, and static tactile displays. (Kaczmarek, 1995)

\section{CONCLUSION}

In this paper, we have presented the concept of the virtual collaborative environment (VCE). A VCE exists when virtual reality and multimedia are combined to create a shared environment or collaborative engineering. We have discussed the problems with such a system such as latency, interface issues and networking issues, and we have presented an architecture for creating such a system which addresses these issues. We have also discussed ways of interacting with the VCE so that users can perform tasks naturally and intuitively. A VCE is an excellent paradigm for collaborative engineering because it allows design and product teams to meet and discuss projects with tools necessary for enhanced communication especially when teams are separated by large geographic regions.

\section{FUTURE WORK}

We will continue to develop and experiment with more visual-coherency techniques such as bending and stretching of objects. We are incorporating camera-based tracking and haptic interfaces into our VCE discussed in section 4. Finally, we plan to perform some user studies to determine if the system could be implemented as a commercial product.

\section{REFERENCES}

Barton, R.J., Newton, G.P., Cross, R.A., and Goettsch, A. (1997) An Architecture for Virtual Collaborative

Environments, submitted for review, 1997 SIGMM.

Brooks, F.P., Ouh-Young, M., Batter, J. and Kilpatrick, P.J. (1990) Project GROPEHaptic Displays for

Scientific Visualization, ACM SIGGRAPH '90, 177-186.

Bukowski, R. and Séquin C. (1995) Object associations: A simple and practical approach to virtual $3 \mathrm{D}$

manipulation, Computer Graphics (1995 Symposium on Interactive 3D Graphics), Apr. 1995.

131-138.

Conner, B. (1997) Providing A Low Latency User Experience In A High Latency Application, Proceedings

of the 3D Symposium on Interactive Graphics, 45-48. 
Kaczmarek, K.A., Bach-y-Rita, P. (1995) Tactile Displays, in Virtual

Environments and Advanced Interface Design, (Eds. W. Barfield, T. A. Furness)

Oxford University Press, London.

Kress, H. and Anderson, B. (1996) HP Shared 3D Viewer - Ein verteiltes 3D

Visualisierungssystem für

Produktdaten,unix/mail, Carl Hanser Verlag München, 329-334.

Pausch, R. (1994) Alice: A Rapid Prototyping System for Virtual Reality. ACM SIGGRAPH Course

Notes 15.1-15.6.

Macedonia, M. (1994) Networking and Communications, in, Virtual Reality

Scientific and Technological

Challenges, (Eds. N. Durlach and A. Mavor) National Academy Press, Ch 10.

Zeleznik, R.C. and Wloka, M. (1994) Interactive Real-Time Motion Blur,

Proceedings of CGI'94: Insight

Through Computer Graphics. 74-85.

Zeleznik, R.C., Herndon, K., Hughes, J. (1996a) Sketch: An Interface for

Sketching 3D Scenes,

Proceedings of SIGGRAPH'96, 163-170.

Zeleznik, R.C. and Wloka, M. (1996b) Interactive Real-Time Motion Blur, Visual

Computer, Springer

Verlag, 1996.

\section{BIOGRAPHY}

Joseph J LaViola Jr.

Joseph J LaViola Jr. is a research intern at Fraunhofer, CRCG. He received his B.S.C.S from Florida Atlantic University in 1996. He is also a Master's student at Brown University studying computer science. His research interests include virtual environments, augmented reality, and human-computer interaction.

He has received numerous awards for scholastic achievement including the Microsoft Senior Achievement award. He is a member of Phi Eta Sigma and Phi Kappa Phi honor societies. He is also a member of ACM SIGGRAPH.

\section{Robert J Barton III, Engineer, Project Leader}

Robert J Barton III received a BS in Mechanical Engineering and an active duty commission from the US Naval Academy, Annapolis, MD. in 1985. Follow on assignments included engineering billets on US Naval Surface ships, instructor duty at the Naval Academy, and systems engineering branch head for the Trident Missile Program of Strategic Systems Programs Office, Sunnyvale, CA. In 1995, Lt Barton completed Masters of Science degrees in Electrical Engineering and Computer Science from Naval Postgraduate School, Monterey, CA. and was awarded the Armed Forces Communications and Electronics Award for academic 
achievement. Areas of research and interest include VLSI High-radix arithmetic circuits, computing the average number of nodes of Ordered Binary Decision Diagrams (OBDDs), and the modeling and analysis of multicast network protocols. Mr. Barton has recently joined the staff of Fraunhofer CRCG, in Providence,RI as an Engineer and Project Leader for the Transatlantic Research and Development Environment (TRADE). Member of IEEE Computer Society and Eta Kappa Nu.

\section{Gregory P. Newton}

Greg Newton is a Researcher and Assistant Project Leader at CRCG. He holds a M.S. in Computer Science from the Georgia Institute of Technology, where he performed a Master's Project on "A Collaborative Environment for the Responsive Workbench". He held a Graduate Research Assistantship at the Georgia Tech Graphics, Visualization \& Usability Center (GVU), where his work supported research in scientific visualization, Distributed Interactive Simulation, terrain visualization, and Simulation-Based Design. He performed several internships at the Naval Research Laboratory, where his work supported research in SimulationBased Design and Command \& Control for the Responsive Workbench.

His current work is in the areas of real-time computer graphics, Distributed Interactive Simulation (DIS) and collaborative virtual reality. His research has been published in the Proceedings of the SPIE Aerospace/Defense Sensing \& Controls Symposium and in IEEE Computer Graphics \& Applications.

$\mathrm{He}$ is a member of the Association of Computer Machinery and the IEEE Computer Society.

\section{Dr. Robert Cross, Scientist and Project Leader}

Dr. Cross is a Scientist and Project Leader at CRCG. He holds a Ph.D. in Computer Science, with a dissertation on "Interactive Visual Realism Using Distributed Rendering." He held an ASEE Post-doctoral fellowship at the Advanced Information Technology Branch of the Naval Research Laboratory, and an Associate Instructorship of Computer Science and a College of Arts and Sciences Fellowship at Indiana University. His research into the problems inherent in real-time volume rendering was published in "Interactive Visualization Methods for Four Dimensions" and "Virtual Reality Performance for Virtual Geometry" in the Proceedings of IEEE Visualization '93 \& '94. In his current work, he seeks solutions to the computationally complex problems of real-time modeling \& simulation and virtual reality, with particular focus on the use of distributed computing to support real-time, high fidelity computer graphics. His work has been published in the Computer Graphik Topics, the Proceedings of IEEE Visualization '93 .- '95 and in a book chapter in Graphics Gems V. He has also presented his work at invited seminars at Brown University and the Naval Research Laboratory as well as at the Virtual Reality Room at ACM SIGGRAPH 
'94. He has also been the recipient of grants from the Franklin Institute, the Office of Naval Research, the American Society for Engineering Education, and the Indiana University College of Arts and Sciences. $\mathrm{He}$ is a member of the Association of Computer Machinery, the IEEE Computer Society, the Mine Warfare Association and the United States Naval Institute. 